CARNETS DE Carnets de géographes

GÉOGRAPHES

$7 \mid 2014$

Les espaces de l'entre-deux

\title{
Les quartiers non-lotis : espaces de l'entre-deux dans la ville burkinabé
}

Ophélie Robineau

\section{(2) OpenEdition}

1 Journals

Édition électronique

URL : http://journals.openedition.org/cdg/478

DOI : $10.4000 /$ cdg.478

ISSN : 2107-7266

Éditeur

UMR 245 - CESSMA

Référence électronique

Ophélie Robineau, «Les quartiers non-lotis : espaces de l'entre-deux dans la ville burkinabé », Carnets de géographes [En ligne], 7 | 2014, mis en ligne le 01 décembre 2014, consulté le 30 avril 2019. URL http://journals.openedition.org/cdg/478; DOI : 10.4000/cdg.478

Ce document a été généré automatiquement le 30 avril 2019

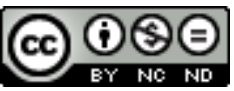

La revue Carnets de géographes est mise à disposition selon les termes de la Licence Creative Commons Attribution - Pas d'Utilisation Commerciale - Pas de Modification 4.0 International. 


\title{
Les quartiers non-lotis : espaces de l'entre-deux dans la ville burkinabé
}

\author{
Ophélie Robineau
}

\section{Introduction}

1 La singularité des dynamiques urbaines africaines ont mis les villes d'Afrique au cœur de nouvelles recherches en géographie (Thornton et Rogerson, 2013). Les formes du développement de ces villes remet en question les théories et les modèles de la géographie urbaine, de la sociologie, de l'anthropologie et de l'aménagement (Myers, 2011). Longtemps étudiées à travers le prisme des villes occidentales, les villes africaines font l'objet d'une nouvelle approche portée par des auteurs anglo-saxons (Myers, ibid.; Simone, 2004; Harrison, 2003) qui soulignent que la plupart des études sur le milieu urbain africain se sont basées sur des modèles américains ou européens, vus comme des modèles universels. Les écritures sur les villes africaines portent souvent " un langage qui est malheureusement enclin à un niveau d'abstraction dans lequel les enjeux de la vie quotidienne sont traduits en plans et politiques qui bien souvent ont très peu de similitudes avec les préoccupations réelles des gens" (Harrison, ibid.: 22). L'analyse des villes africaines, de leur «alter-modernité» (Chenal et al., 2009), nécessite ainsi de leur reconnaître des particularités qui échappent aux cadres d'analyses adaptés aux villes du Nord: ces auteurs parlent d'une "alter-modernité qui n'est plus le fruit de métissages entre un modèle blanc et colonial et des pratiques noires et indigènes, mais qui résulte d'un autre "chemin ", vernaculaire peut-être, définissant ses propres règles, affirmant avec résolution sa modernité autre» (ibid.). A ce titre, Simone (ibid.) explique comment la ville africaine pourrait mieux se comprendre à travers les notions d'informalité et d'invisibilité. Ces deux notions se complètent. En effet, l'informalité renvoie à l'importance de l'économie informelle, et à la manière dont elle imprègne les dynamiques urbaines et caractérise les villes africaines depuis les pratiques citadines jusqu'à la gouvernance urbaine. Ce que certains auteurs nomment " la crise urbaine ", due notamment à la désorganisation partielle ou totale de l'encadrement étatique, a longtemps été amortie par le dynamisme des habitants des 
villes : on observe un développement important du secteur informel, à la fois dans l'habitat et dans les activités économiques (Dubresson et Raison, 1998). L'invisibilité quant à elle caractérise les pratiques quotidiennes des urbains, informelles, conduites dans des quartiers "illégaux », dans des interstices urbains, invisibles aux yeux du secteur formel, et invisibles dans les documents officiels tout comme dans la planification urbaine. Les auteurs incitent ainsi à dépasser les cadres d'analyse construits à l'aune d'expériences extérieures et à aborder la modernité des villes africaines non pas dans la dualité et l'opposition des termes (légal/illégal, moderne/traditionnel) mais dans leur perméabilité (Bertrand, 1998).

2 Les études urbaines conduites sur les villes africaines mettent largement en évidence l'importance des habitats «illégaux» dans l'espace urbain. L'urbanisation est la transformation qui a le plus affecté le continent africain depuis les Indépendances (Losch et al., 2013). La population totale a triplé entre 1950 et 1995, mais le nombre de citadins a été multiplié par neuf (19,6 millions à 176,6 millions). Le taux croissance des populations urbaines en Afrique de l'Ouest est en moyenne de $5 \%$ par an (ONU, 2009). Dans ce contexte, la production de logements « légaux » ne suffit pas à accueillir une population urbaine toujours plus importante et bon nombre de citadins en situation précaire vont habiter en périphérie des villes, dans des quartiers d'habitat dit «spontanés » ou « informels » (Vernière, 1973). Souvent décrits comme des bidonvilles (Davis, 2005) ces quartiers spontanés font parfois plutôt penser à de l'habitat rural aux portes des villes; le terme de bidonville semble alors trop fort pour qualifier l'habitat informel. Quel que soit le visage de ces quartiers spontanés ou leur dénomination, ils abritent une part importante de la totalité de la population de certaines villes ; l'ONU-Habitat a estimé en 2008 que près de $50 \%$ des urbains africains vivent dans des quartiers spontanés (ONUHabitat, 2008). Mais malgré l'importance de ces quartiers, ils n'apparaissent pas sur les plans des villes, ils sont le «blanc » des cartes (Vasset, 2007) et personne ne pourrait trouver un plan pour s'y repérer (Myers, ibid.).

3 Cet article s'intéresse à un type particulier d'habitat spontané : les quartiers « non-lotis " du Burkina Faso. En débutant un travail de recherche de terrain à Bobo-Dioulasso (500.000 habitants), la seconde ville du pays, ces « blancs » des cartes nous ont interpellé. Localisés en bordure de l'espace bâti légal, ou parfois même insérés en son sein, aucune information n'apparaissait sur ces espaces dans les documents cartographiques. Mais audelà des cartes, la réalité sur le terrain est toute autre. Au Burkina Faso, les franges urbaines des villes sont de vastes zones d'habitat qualifiées d'illégales, de spontanées, d'informelles ou plus couramment de «non-lotis». Le terme de «non-loti» vient en opposition avec celui de quartier « loti » légal, aménagé et viabilisé dont l'organisation en damier découle des politiques d'aménagement coloniales (Ouattara, 2004). A BoboDioulasso, ces zones non-loties couvrent plus de 4.000 ha (la ville « légale » couvre 86.000 ha). A la fois considérés comme faisant partie de la ville mais présentant un habitat proche de l'habitat rural, les «non-lotis » semblent être des espaces de l'entre deux, à l'interface entre l'urbain et le rural.

4 Quelle réalité couvre la présence de ces zones d'habitat non-loti « invisibles ", à la fois rattachés à la ville mais de configuration plutôt rurale? Quelle est leur origine et leur place dans le processus d'urbanisation de cette ville africaine? Pour répondre à ces questions, nous revenons tout d'abord sur l'origine des quartiers non-lotis, avant d'analyser la place qu'ils occupent dans le processus d'urbanisation actuel et les pratiques des acteurs qui contribuent à en faire des espaces de l'entre deux singuliers. Nous 
discuterons alors la place qu'occupent ces quartiers dans la construction de la ville africaine, en tant qu'espace transitoire ou temporaire dans le processus d'urbanisation.

\section{Aux origines des non-lotis}

\section{De la naissance de la ville au lotissement colonial autoritaire}

En Afrique occidentale, les échanges marchands ont parfois fait de l'accessibilité le point fort d'une ville (Coquery-Vidrovitch, 2006). La ville de Bobo-Dioulasso trouve ses origines dans l'installation d'agriculteurs d'ethnie Bobo avant le $15^{\text {sème }}$ siècle. Puis elle s'est développée à partir du $15^{\text {ème }}$ siècle dans un contexte géographique favorable, au carrefour de plusieurs axes de commerce d'Afrique de l'Ouest, à mi-chemin entre le Sud forestier et ses produits (or, cola) et le Nord sahélien et saharien (sel, bétail). Son véritable essor date des $18^{\text {ème }}$ et $19^{\text {ème }}$ siècles, avec le développement des échanges marchands. Selon Fourchard (2001) c'est à cette période qu'il est permis d'affirmer qu'elle accéda au rang de ville : il existait déjà un surplus de production agricole susceptible de nourrir les nonagriculteurs, ainsi qu'un groupe de commerçants et un pouvoir politique capable d'imposer la production et la circulation des vivres.

Devant l'intérêt commercial que représentait la situation géographique de BoboDioulasso, les colons français s'y installèrent à la fin du $19^{\text {ème }}$ siècle. Jusqu'aux années 1920, les infrastructures coloniales se surimposaient à la ville africaine. Bobo-Dioulasso restait une ville africaine entourant un petit poste administratif colonial. C'est avec le premier plan de lotissement, mis en place à partir de 1926, qu'il y eut une rupture dans le processus d'urbanisation afin de répondre à l'idéal colonial. Avec l'arrivée importante d'européens pendant les années 1920, l'administration mit en place une règlementation contraignante pour protéger la santé et le mode de vie des européens. La nouvelle organisation de la ville que les colons allaient mettre en place entre 1926 et 1929 répondait à des principes hygiénistes, basés sur une ségrégation en fonction de l'origine géographique ou ethnique des habitants (Fourchard, ibid.; Goerg et Huetz de Lemps, 2012).

Le plan de lotissement mit fin à la densification des quartiers précoloniaux Bobo par le tracé autoritaire de grandes voies et l'aménagement de nouvelles zones d'extension urbaine. Le damier, utilisé dans bon nombre de villes de cette période coloniale, fut aussi le modèle mis en place à Bobo-Dioulasso : le procédé de lotissement était en effet un outil de planification qui véhiculait des valeurs esthétiques, trouvait des justifications hygiénistes et symbolisait l'ordre tout en permettant un hiérarchisation de l'espace (Goerg et Huetz de Lemps, ibid.). A l'exception de quelques quartiers, l'ensemble des quartiers précoloniaux fut loti. Les premiers plans de lotissement marquaient le début d'une classification de ce qui relevait du légal et du formel - à travers une officialisation écrite de l'occupation des terrains dans les zones loties -, et de l'illégal et de l'informel, non officialisé, comme les droits de propriétés coutumiers.

Ce modèle d'aménagement urbain se modifia peu après l'Indépendance. Aujourd'hui il est toujours d'actualité de faire des lotissements et de parcelliser en damier pour organiser la ville (Robineau, 2013 ; Jaglin, 1995). Le lotissement des quartiers est basé sur la création de quartiers ex-nihilo à partir d'un déguerpissement des familles cultivant ou habitant les terres à urbaniser. 


\section{La post-Indépendance : croissance urbaine rapide et carence de logements}

Comme la majorité des villes africaines, la ville de Bobo-Dioulasso connut une forte croissance démographique après l'Indépendance. Alors que les colons s'étaient attachés à privilégier l'accès à la ville à la seule main-d'œuvre nécessaire au fonctionnement de leurs services et à une poignée de commerçants, les villes devenaient à l'Indépendance un nouveau lieu d'opportunités pour les habitants du monde rural (Fournet-Guérin, 2011). Entre 1960 et 1985, la population de Bobo-Dioulasso passa de 52.000 à 230.000 habitants (INSD, 2006). Aujourd'hui, la ville compte près de 500.000 habitants et les prévisions annoncent une croissance démographique encore soutenue pour les décennies à venir (Commune de Bobo-Dioulasso, 2007).

10 Cette forte augmentation de la population urbaine entraîna un retard croissant de l'offre de logements par rapport à la demande (Osmont, 1995). Beaucoup de familles allaient construire sans autorisation administrative et de façon clandestine des maisons en banco ${ }^{1}$ (figure 1) à la limite des quartiers lotis, sur des terres qu'elles achetaient à des autochtones. L'accroissement spatial régulier de la ville était principalement le fait de l'extension des zones d'habitat spontané (appelées aussi les zones « non-loties »).

Figure 1. Maisons en banco d'une zone non-lotie. L'antenne de télévision témoigne d'un accès à l'électricité via des raccordements illégaux au réseau électrique de la zone lotie voisine

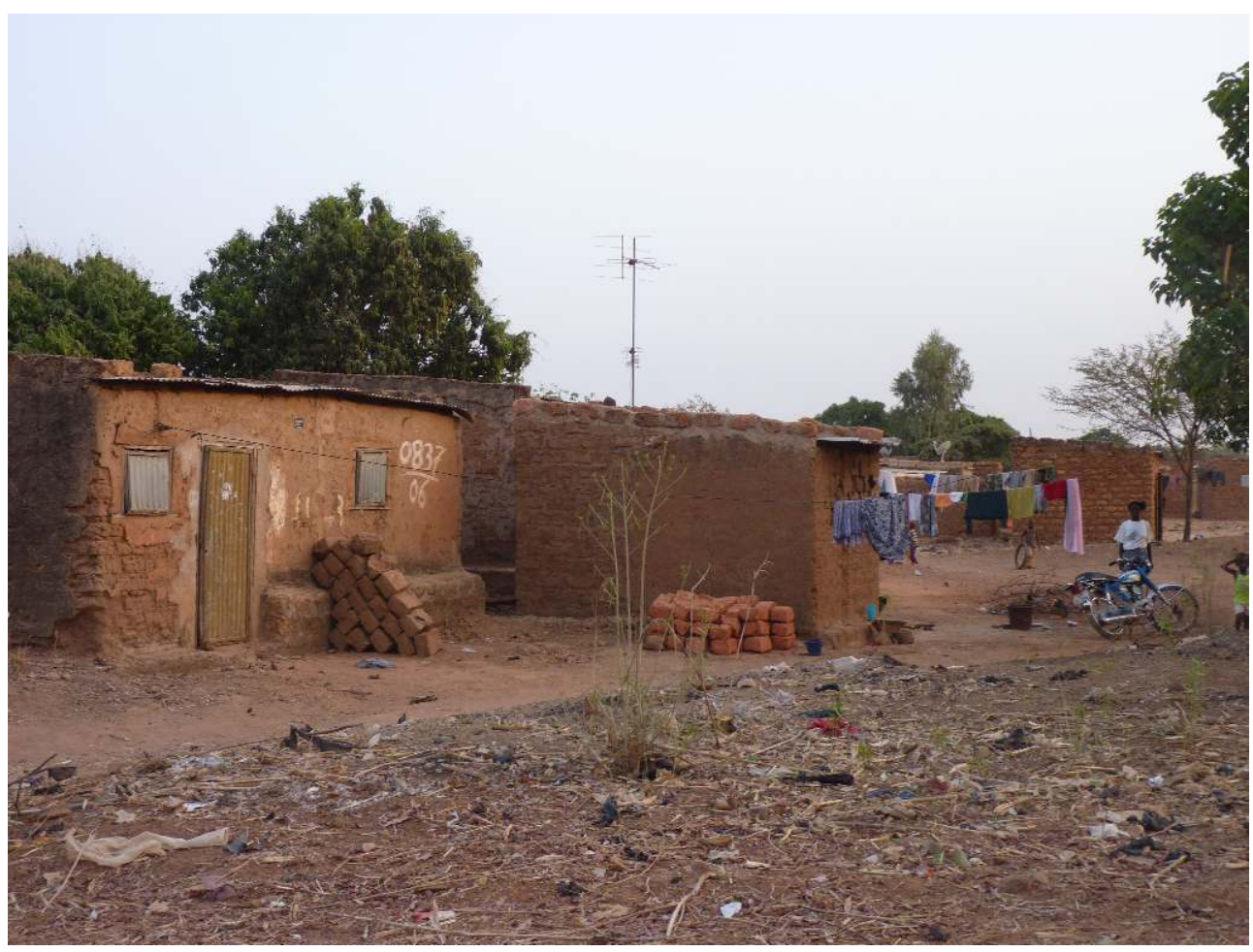

Cliché : Robineau O. 2012

Pour remédier à l'accroissement de ces zones non-loties, un vaste programme de lotissement fut mis en place en 1985, organisé dans le cadre du Programme Populaire de Développement : ce programme était symbolisé par le slogan « une famille, un toit ». De 1983 à 1990, le milieu urbain connut ainsi l'émergence d'une politique volontariste qui 
afficha comme ambition la prise en charge de l'accès au logement pour le plus grand nombre (IAGU et ONU-Habitat, 2005). Des vagues de lotissement se succédèrent de 1984 à nos jours (figure 2) dans le but de résorber l'habitat illégal (les "non-lotis ») et de maîtriser la croissance spatiale urbaine. Afin de faciliter la mise en place de ce programme, la loi sur la Réorganisation Agraire et Foncière (RAF) fut votée pour abolir toutes les entraves à la promotion du monde paysan rural et des zones urbaines. Un Domaine Foncier National (DFN) fut créé, constitué de toutes les terres situées dans les limites du territoire national : le DFN fut déclaré propriété exclusive de l'Etat, inaliénable, imprescriptible et insaisissable. Ceci mit hors jeu les propriétaires fonciers coutumiers qui étaient désormais expropriés des zones à urbaniser. Mais les lotissements successifs ne furent pas suffisants pour éviter le développement des zones non-loties, qui entourent toujours aujourd'hui les zones loties.

Figure 2. Expansion de l'espace bâti loti et non-loti entre 1981 et 2012.

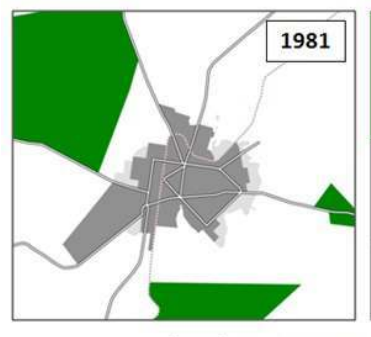

Zone bâtie lotie

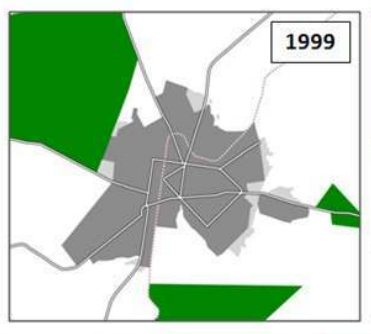

Zone bâtie non-lotie

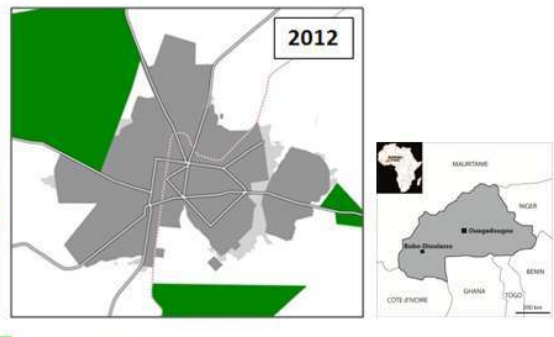

Forêtclassée

Réalisation : Robineau O. 2012 identifiables par leurs formes spatiales (figure 3). Ils correspondent à trois modes d'urbanisation qui s'enchevêtrent et qui se retrouvent couramment dans les villes d'Afrique (Yapi-Diahou, 2007) : avec adduction d'eau potable et d'électricité et un tracé rectiligne des rues. L'héritage colonial a laissé une armature urbaine très structurée avec de larges avenues bordées de grands arbres. Cette ville « légale » se construit selon les règles officielles. Elle est sous le périmètre d'intervention du pouvoir public:

- Les zones non loties : leur organisation spatiale ne suit pas des formes géométriques. Il s'agit de la ville "illégale et informelle», créée en dehors de la procédure officielle de production d'espaces bâtis. Dans les zones non-loties, l'habitat n'est pas planifié, il n'y a pas d'adduction d'eau ni d'électricité, pas de rues bitumées, et il y a une absence d'officialisation de l'occupation des terrains. L'habitat est majoritairement construit en terre. On retrouve les zones non-loties presque tout autour de la ville, en limite des zones loties ;

- Les zones d'ancien habitat rural qui ont été englobées par la ville. Il s'agit de quartiers précoloniaux qui gardent leur architecture d'origine ${ }^{2}$. Ces zones d'habitat ne sont pas illégales, mais ne jouissent pas d'un statut légal. Certains de ces quartiers historiques ont été restructurés (organisation orthogonale, adduction d'eau et d'électricité) et intégrés dans la zone lotie légale. D'autres ont gardé leur organisation en habitat rural car les autorités traditionnelles refusent purement et simplement le lotissement qui engendrerait une restructuration sociale et spatiale de leur quartier. 
Figure 3. Vues aériennes d'une zone lotie (1), non-lotie (2) et d'ancien habitat rural (3)
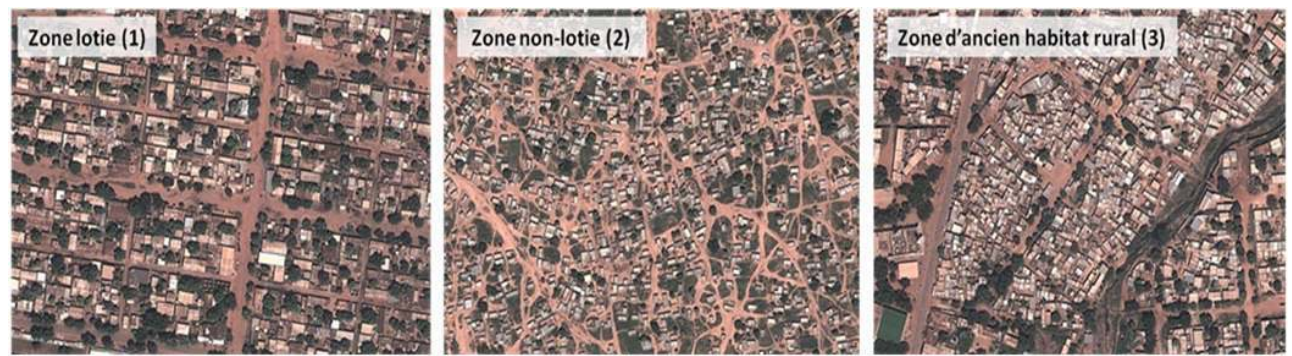

Source : images Google Earth 2013

Les zones non-loties et d'ancien habitat rural forment des discontinuités spatiales dans la ville : la densité élevée de l'habitat et l'organisation non-géométrique font rupture avec le lotissement orthogonal. Il s'agit maintenant de comprendre les pratiques et les stratégies des acteurs qui sont à l'œuvre dans les zones non-loties et de saisir la façon dont ces zones s'inscrivent spatialement et temporellement dans le processus d'urbanisation.

\section{Les zones non-loties : des zones informelles au cœur du processus d'urbanisation}

15 Les zones non loties sont des zones entre l'urbain et le rural. L'architecture des maisons et l'organisation des habitations y est plus proche du village que de la ville. Aussi, la gestion foncière se trouve au croisement entre les lois appliquées en milieu urbain et la gestion coutumière appliqué en milieu rural. Les zones non-loties sont à la fois la conséquence du flou juridique qui existe autour de la gestion foncière en frange urbaine, et l'illustration de la difficulté qu'ont les autorités urbaines à produire assez de logements pour l'ensemble des familles qui recherchent un toit.

\section{Un sas pour accéder à un logement... et à la ville}

Se loger est un défi pour les familles et acquérir une parcelle lotie est coûteux. Alors que certaines familles ont les moyens financiers de choisir leur logement dans les quartiers lotis, d'autres sont dans l'incapacité financière d'acheter un terrain ou une maison dans ces mêmes quartiers.

Le prix d'une parcelle lotie - d'une taille standard d'environ $300 \mathrm{~m}^{2}$ - avoisine $900.000 \mathrm{FCFA}^{3}$ (soit $3.000 \mathrm{FCFA} / \mathrm{m}^{2}$, auxquels il faut ajouter la construction de la maison). La solution pour les familles qui n'ont pas les moyens d'acheter une telle parcelle est donc de louer une petite maison, ou bien d'acquérir une parcelle en frange urbaine, en zone non-lotie, pour y construire une maison en banco. Dans cette zone les prix sont plus abordables qu'en zone lotie car les parcelles achetées sont de taille réduite - même si le prix au $\mathrm{m}^{2}$ reste élevé à cause de la demande qui existe pour ces terres (environ 80.000 FCFA pour des parcelles de $50 \mathrm{~m}^{2}$ en 2011, soit environ 1.600 FCFA le $\mathrm{m}^{2}$ ). L'accès au terrain en zone non-lotie se fait à travers des négociations avec les propriétaires coutumiers des villages Bobo mais cette procédure ne bénéficie d'aucune reconnaissance formelle de la part des autorités urbaines. 
les habitants des zones non-loties, vivre dans le non-loti ne signifie pas réellement être en ville. La présence d'écoles et de lieux de cultes ${ }^{4}$ au sein de ces zones ne sont pas des critères suffisants pour se sentir citadin. Les zones non-loties sont un " entre-deux » : les familles qui y vivent se considèrent être aux portes de la ville, en attendent de pouvoir y être pleinement intégrés, comme en témoigne Michel $\mathrm{S}$, ancien habitant d'une zone non-lotie.

«Au non-loti, je n'habitais pas en ville. Il n'y avait pas d'eau, pas d'électricité. Ici maintenant oui, je suis en ville. Il y a l'eau, il y a l'électricité et tu peux bien construire. Je me sens mieux dans le loti car je suis tranquille dans ma parcelle. Au non-loti ce n'est pas reconnu officiellement. Du jour au lendemain on peut te demander de partir, c'est pas facile» (Michel S., ancien habitant d'une zone nonlotie)

Les habitants des zones non-loties combinent des activités dans la ville « légale » et dans la ville «illégale». Ils tentent de recréer le mode de vie de leur village d'origine et mènent une existence leur permettant à la fois de tirer partie de l'informalité de ces zones (en y développent des cultures hautes ${ }^{5}$ ou des élevages de porcs, qui sont des activités interdites dans les zones loties), tout en bénéficiant des services urbains à proximité (électricité via des raccords illégaux au réseau le plus proche, eau potable, écoles, commerces). Les zones non-loties sont donc une frange urbaine à la fois spatiale et sociale et reflètent aujourd'hui un nouveau type un nouveau type de relation à la ville hybridant ruralité et urbanité. Cependant, l'incertitude quant à la pérennité de cette situation caractérise le ressenti des habitants de ces quartiers.

Devant la difficulté financière et logistique qu'a parfois la ville à créer des lotissements, certaines zones non-loties ont plus de vingt ans. Mais leur l'ancienneté n'enlève en rien le caractère temporaire qu'elles ont dans le processus d'urbanisation. En effet, la création de logements «légaux » par du lotissement se fait soit sur des champs, soit sur des zones d'habitat spontané où les maisons sont alors détruites. Lors d'opérations de lotissements, la mairie établit une liste d'attributaires pour les parcelles loties en fonction de critères prédéfinis. Des agents municipaux effectuent le recensement des familles qui résident dans les zones non-loties. Si un logement est inhabité, il n'apparaît pas dans le recensement. Pour éviter de manquer la venue des agents en cas d'absence et de perdre ainsi la chance d'être recensées, des familles affichent un panneau sur leur maison indiquant le nom, la date de naissance, le numéro d'identité et parfois le numéro de téléphone du chef de famille (figure 4 ci-dessous). Le recensement concerne aussi les agriculteurs propriétaires coutumiers des terres à lotir. 
Figure 4. Panneau indiquant l'identité du propriétaire d'une maison dans un quartier non-loti
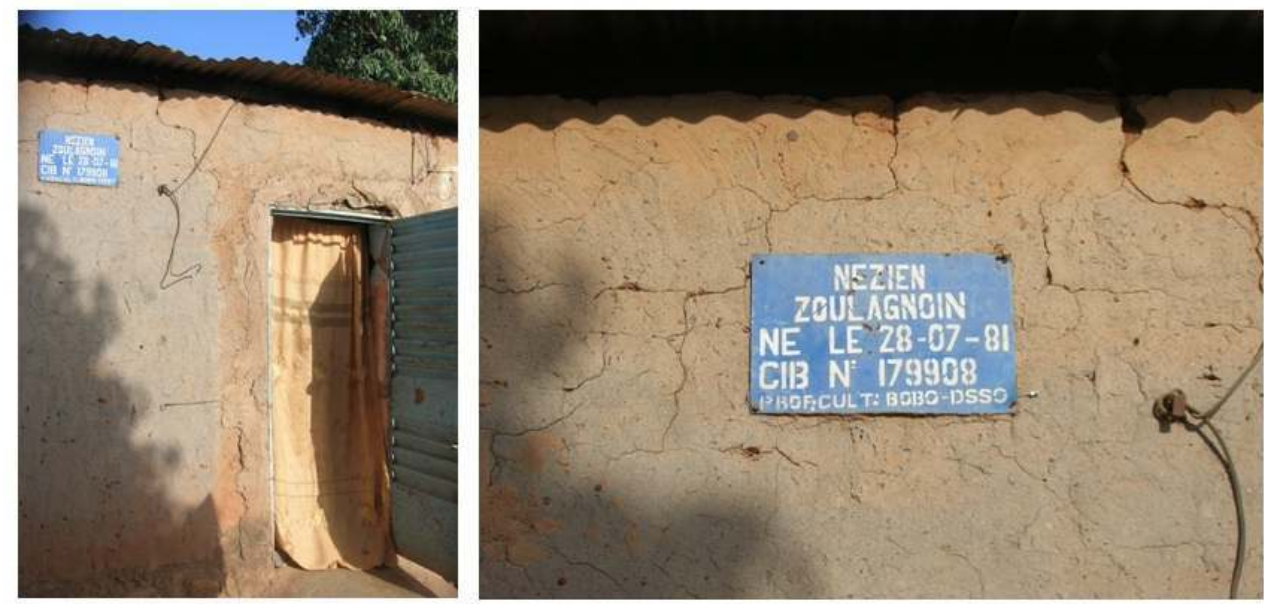

Cliché : Robineau O. 2012. de la construction d'une zone lotie. Il s'agit 1) de familles d'agriculteurs expropriés dans le cadre du lotissement (chaque propriétaire coutumier est compensé de deux parcelles constructibles par hectare de champ exproprié). Bien qu'aucun texte de loi ne prévoie la compensation des propriétaires coutumiers expropriés, l'Etat préfère ménager le système coutumier traditionnel en procédant à une compensation en parcelles constructibles afin d'éviter les conflits (Hilgers, 2009) ; 2) de familles qui résidaient dans la zone non-lotie (les «déguerpis ») et 3) de familles qui ont fait la demande de parcelles auprès de la mairie. Si le nombre de bénéficiaires recensés excède le nombre de parcelles constructibles disponibles, un classement est effectué en fonction des caractéristiques socio-économiques des ménages. Dans ce classement, les propriétaires coutumiers sont prioritaires. Ensuite, il s'agit des ménages expropriés et de ceux qui ne possèdent pas déjà de parcelles en zone lotie.

Une fois la liste de bénéficiaires arrêtée, les parcelles constructibles sont attribuées. Etre attributaire d'une parcelle lors d'une opération de lotissement est ainsi le moyen le plus économique d'avoir accès à un logement légal. C'est pourquoi les zones non-loties restent accolées aux zones loties, et qu'on ne retrouve peu ou pas de zones non-loties construites dans les zones où la ville a théoriquement freiné l'expansion des constructions depuis plusieurs années: installer sa maison au plus proche du loti permet d'augmenter ses chances d'être inclus dans le prochain lotissement (qui peut être réalisé au cours de la décennie suivante, selon les échéances prévues dans le plan d'aménagement de la ville). Le non-loti représente alors un entre-deux entre la précarité et la stabilité : plus la situation foncière est précaire (au vu de l'imminence d'un lotissement), plus l'objectif d'atteindre une situation d'habitat pérenne est grande.

\section{Un entre-deux qui favorise la spéculation foncière}

Les habitants de Bobo-Dioulasso sont conscients de l'intérêt de passer d'une propriété en zone non-lotie à une propriété dans un quartier loti. La valeur de la parcelle augmente considérablement et, selon les quartiers, le prix au mètre carré peut doubler ou tripler. L'espoir d'être attributaire d'une parcelle lors d'un lotissement fait que l'on retrouve 
deux types de propriétaires d'habitations dans les zones non-loties :

1) des familles en situation financière précaire qui construisent dans le non-loti car l'acquisition de parcelle y est plus facile est moins onéreuse; «Si on arrive à avoir une parcelle dans le loti alors on pourra dire qu'on habite en ville » (Rosalie $O$, habitante du nonloti).

2) les familles des classes moyennes qui attendent que le lotissement soit réalisé : elles spéculent en acquérant une "parcelle-alibi » dans une zone non-lotie (terme employé par Jaglin, 1995) et en y construisant une maison.

Jaglin (ibid.) a identifié les mêmes types de propriétaires d'habitations dans les zones nonloties à Ouagadougou où, tout comme à Bobo-Dioulasso, les familles espèrent pouvoir un jour bénéficier d'une parcelle en zone lotie (Ouattara, 2004).

Mais le processus d'attribution des parcelles dans les futures zones loties - qui permet à moindre frais d'accéder à la propriété de biens immobiliers - engendre des contournements du processus formel de la part des élus. Dans les faits, les critères d'attribution ne sont que partiellement respectés et les opérations d'attribution de parcelles cachent des négociations dans lesquelles des personnes influentes en profitent pour augmenter leur patrimoine foncier. Leurs liens avec des élus ou des personnes proches des élus leur permettent de pouvoir apparaître sur la liste des bénéficiaires de parcelles constructibles. Ceci fait parfois éclater des scandales ${ }^{6}$, et il n'est pas rare de voir des manifestations en ville contre la procédure d'attribution des lots. De plus, selon la superficie de la zone non-lotie à détruire et le nombre d'agriculteurs possédant des champs sur la zone à lotir, gérer l'attribution de parcelles constructibles peut parfois relever du casse-tête. C'est notamment le cas du lotissement prévu après 2010 sur une zone non-lotie au sud de la ville, où 9.000 concessions ont été recensées dans le non-loti (sans compter des agriculteurs possédant des champs dans la zone à lotir) alors qu'il y a seulement 4.600 parcelles prévues dans la zone à urbaniser. Dans ce cas de figure, seulement une partie des familles qui habitent la zone non-lotie bénéficie d'une parcelle lors de leur expropriation. Finalement, les résidents de ces espaces de l'entre-deux sont ceux dont la situation est la plus incertaine lorsque que les zones non-loties sont détruites : les plus chanceux accèdent enfin à la ville « légale » en se voyant attribuer une parcelle constructible, et les autres doivent trouver un nouvel espace pour reconstruire leur habitat aux portes de la ville. Ils s'en retournent alors à une situation d'habitat précaire et transitoire, en nourrissant de nouveau l'espoir d'être bénéficiaire d'une parcelle légale lors d'un prochain lotissement.

\section{Discussion et conclusion}

Que ce soit pour y habiter ou pour spéculer, le caractère informel, précaire, temporaire et transitoire des zones non-loties les place au cœur d'un ensemble de stratégies développées par tout un panel d'acteurs. Elles sont, aux yeux des familles qui les habitent, un sas pour accéder à la ville qui permet de passer progressivement, et à moindre du coût, du milieu rural au non-loti, pour accéder enfin à la ville légale. Elles sont aussi l'espace des spéculations foncières pour les classes moyennes, qui ne bénéficient pas de relations personnelles dans les hautes sphères de la ville pour contourner les procédures d'attribution, mais qui tentent leur chance avec des parcelles-alibi. Bien qu'invisibles sur les cartes, les zones non-loties sont pourtant visibles aux yeux des autorités et des 
fonctionnaires de la ville qui tirent partie de l'informalité de ces espaces pour se générer des rentes foncières en contournant les procédures d'attribution des parcelles loties.

A l'inverse de nombreuses villes du Sud où les quartiers spontanés perdurent dans le temps, jusqu'à se densifier et parfois devenir des quartiers populaires faisant partie intégrante de la ville (Petropoulou 2007), les zones non-loties du Burkina Faso ont un caractère transitoire dans le processus d'urbanisation, où elles sont vouées à être complètement détruites à court ou moyen terme pour céder la place à des zones d'habitat « légal ». La dénomination de zones «non-loties» reflète alors ce caractère transitoire: elles «ne sont pas» le légal, ni la ville telle que les documents de planification la prévoient. Elles ne sont plus le rural, mais ne sont pas encore tout à fait la ville. Elles sont un entre-deux de transition et c'est à travers cette singularité qu'elles existent. Elles sont à la fois un entre deux socio-spatial entre le rural et l'urbain, un entre-deux temporel entre l'étape de l'urbanisation informelle puis celle de l'urbanisation formelle, et un entre-deux juridique et politique entre la procédure informelle d'accès au logement dans le non-loti et la procédure formelle d'attribution des parcelles loties.

La permanence des zones loties dans le paysage urbain amène à repenser la ville autrement et imaginer les zones loties et non-loties non pas comme des espaces aux dynamiques opposées, mais comme des espaces aux dynamiques complémentaires, ou s'enchevêtrent formalité et informalité. Nuttall et Mbembe (2008, p. 9) soulignent que dans les villes africaines "l'informel n'est pas en dehors du formel", ils fonctionnent ensemble et c'est leur combinaison qui participe à produire des formes et des économies urbaines particulières. En effet, dans les villes africaines, le légal et l'illégal, le moderne et le traditionnel, le formel et l'informel, se côtoient, se combinent et se chevauchent et engendrent des formes d'organisation de l'espace et des rapports sociaux qui sortent des cadres classiques d'analyse appliqués dans les villes du Nord. Les zones non-loties sont à l'image de cette combinaison entre le formel et l'informel. Loin d'être en opposition avec la ville "formelle» et "légale», elles font partie d'un processus d'ensemble d'urbanisation de Bobo-Dioulasso, où se côtoient des pratiques formelles et informelles d'accès à l'espace. Ceci éclaire sur la façon dont les pratiques habitantes façonnent l'espace urbain au-delà de l'application formelle des politiques planificatrices et questionne ainsi la place à donner aux processus informels dans la planification urbaine africaine.

\section{NOTES}

1. Maisons construites en briques crues séchées assemblées par un mortier en terre

2. La ville s'est créée et étendue à partir de ces premiers quartiers. Il s'agissait de villages dont l'agglomération et l'augmentation de la population à peu à peu engendré la création d'un centre urbain.

3. 900.000 FCFA équivalent à $1372 €$. Le salaire minimum garanti au Burkina Faso est de 30.684 FCFA/mois, soit $46,8 € /$ mois (chiffre de l'Institut National de Statistique et de Démographie du Burkina Faso, www.insd.bf). Par ailleurs, deux tiers des dépenses est destiné à 
l'alimentation, l'habillement et la santé (données de l'INSD), ce qui fait que la famille ne dispose plus que de 10.000 FCFA/mois pour se loger.

4. Dans certaines zones, des espaces prévus pour les écoles sont délimités bien avant que le lotissement ne soit effectué. Sur ces espaces, aucune habitation n'est installée et les écoles sont construites et fonctionnelles alors même que le secteur n'est pas encore loti.

5. Le maïs, le mil et le sorgho sont définis comme des cultures hautes. Ces cultures sont interdites au sein de la ville pour des raisons sécuritaires : leur taille pourrait permettre à des bandits de se camoufler.

6. Voir à ce propos l'article paru dans Faso.net le 12 août 2012 «Scandale foncier à Bobo : le maire de Konsa octroie plus de $8.000 \mathrm{~m}^{2}$ à son papa Barro Djanguinaba » http://www.lefaso.net/ spip.php?article49579

\section{RÉSUMÉS}

Face à la croissance rapide des villes africaines, les Etats n'arrivent pas à produire suffisamment de logements pour l'ensemble de la population et les familles les plus précaires vont vivre en périphérie des villes, dans des quartiers dits "spontanés». Construits en marge du processus d'urbanisation formel et légal, ces quartiers sont souvent invisibles dans les documents d'urbanisme, même s'ils abritent parfois la majorité de la population urbaine. Au Burkina Faso, ces quartiers sont appelés les «non-lotis ». Malgré leur informalité et leur invisibilité, ces quartiers de l'entre deux - entre deux social, spatial et juridique - font partie intégrante du processus d'urbanisation de la ville et matérialisent un nouveau type d'urbanité, à mi-chemin entre le rural et l'urbain et entre le « formel » et « l'informel ».

Faced with the rapid growth of African cities, states fail to produce enough housing for the entire population and the most vulnerable families go living in the outskirts of cities, in areas so-called "spontaneous settlements". Built out of the formal and legal process of urbanization, these areas are often invisible in the planning documents, although they may harbor the majority of the urban population. In Burkina Faso, these districts are called "non-lotis". Despite their informality and their invisibility, these neighborhoods "in between" - social, spatial and legal "in between" are an integral part of the city's urbanization process and materialize a new type of urbanity, halfway between rural and urban and between "formal" and "informal".

\section{INDEX}

Thèmes : Carnets de recherches

Mots-clés : Burkina Faso, informel, urbanité, invisibilité

\section{AUTEUR}

OPHÉLIE ROBINEAU 